\title{
A Systematic Review of Peripheral and Central Nervous System Involvement of Rheumatoid Arthritis, Systemic Lupus Erythematosus, Primary Sjögren's Syndrome, and Associated Immunological Profiles
}

\author{
Anastasia Bougea, Evangelos Anagnostou, Giatas Konstantinos, Paraskevas George, \\ Nikolaos Triantafyllou, and Evangelia Kararizou
}

1st Department of Neurology, University of Athens Medical School, Eginition Hospital, Athens, Greece

Correspondence should be addressed to Anastasia Bougea; annita139@yahoo.gr

Received 30 June 2015; Revised 21 October 2015; Accepted 29 October 2015

Academic Editor: Gabriel J. Tobón

Copyright (C) 2015 Anastasia Bougea et al. This is an open access article distributed under the Creative Commons Attribution License, which permits unrestricted use, distribution, and reproduction in any medium, provided the original work is properly cited.

Both central (CNS) and peripheral nervous system (PNS) complications are frequent and varied in connective tissue diseases. A systematic review was conducted between 1989 and 2014 in the databases Medline, Scopus, and Cochrane Library using the search terms, peripheral and central nervous complications and immunological profiles, to identify studies in specific connective tissue disorders such as rheumatoid arthritis, systemic lupus erythematosus, and primary Sjögren's syndrome. A total of 675 references were identified, of which 118 were selected for detailed analysis and 22 were included in the final review with a total of 2338 participants. Our search focused only on studies upon connective tissue disorders such as rheumatoid arthritis, systemic lupus erythematosus, and primary Sjögren's syndrome associated with seroimmunological data. The reported prevalence of CNS involvement ranges from 9 to $92 \%$ across the reported studies. However, the association between CNS and PNS manifestations and seroimmunological profiles remains controversial. To date, no laboratory test has been shown as pathognomonic neither for CNS nor for PNS involvement.

\section{Introduction}

Connective tissue disorders are chronic inflammatory autoimmune diseases driven by an antibody or T-cell response directed against a self-antigen antibody affecting muscle, joints, and skin such as rheumatoid arthritis (RA), systemic lupus erythematosus (SLE), primary Sjögren's syndrome (pSS), and systemic sclerosis [1-3]. Both immune mediated changes in the vasculature of the vessels walls, as a hallmark of vasculitis, may be associated with central nervous system (CNS) and peripheral nervous system (PNS) symptoms. The vascular injury may be related to the presence of antibodies most commonly, but not limited to, SS or a related profile of autoantibodies including antinuclear antibody (ANA), extractable nuclear antigen antibodies (SSA/SSB), rheumatoid factor (RF), anticardiolipin antibodies (ACA), cryoglobulins, and anti-double-stranded DNA antibody (A-ds
DNA) [4] (Table 1). Anti-ribosomal P antibodies have been associated with CNS SLE disease [5].

To our knowledge this is the first systematic review focused in both most common CNS and PNS complications of RA, SLE, and pSS emphasizing the associated immunological features of these specific connective tissue.

\section{Methods}

2.1. Eligibility Criteria and Source Selection. This is a systematic review of literature, based on the PRISMA guidelines. We search for relevant studies in English in the following databases, from the emergence of the condition to October 2014: Medline (from 1989 to 2014), Scopus (from 1983 to 2012), and Cochrane Library (from 1993 to 
TABLE 1: Most frequent autoantibodies in nervous system involvement in connective tissue diseases SLE, SS, and RA.

\begin{tabular}{ll}
\hline $\begin{array}{l}\text { Connective tissue } \\
\text { diseases }\end{array}$ & $\begin{array}{l}\text { Autoantibodies (\% of positive patients, } \\
\text { clinical association) }\end{array}$ \\
\hline & ANA \\
& Anti-dsDNA (nephritis, disease activity) \\
& Anti-ribosomal P (disease activity, NPSLE) \\
& Anti-Sm (highly specific for SLE) \\
Anti-Ro (DD Sjögren's syndrome) \\
Anti-La (DD Sjögren's syndrome) \\
Antiphospholipid (thrombosis) \\
& AQP-4 (overlap NMO spectrum) \\
& ANA \\
& Anti-Ro (DD SLE) \\
& Anti-La (DD SLE) \\
& RF (lymphoma) \\
SS & Aryoglobulins (vasculitis, lymphoma) \\
& AQP-4 (overlap NMO spectrum) \\
\hline RA & ANA
\end{tabular}

SLE: systemic lupus erythematosus, NP: neuropsychiatric, SS: Sjögren's syndrome, RA: rheumatoid arthritis, ANA: antinuclear antibodies, AntiSm: anti-Smith, Anti-Ro: Ro antigen, Anti-La: lupus anticoagulant, antidsDNA: anti-double-stranded DNA, RF: rheumatoid factor, DD: differential diagnosis, AQP-4: Aquaporin, and NMO: Neuromyelitis optica.

2014). The keywords used for the study were the following: "peripheral nervous and central nervous manifestations," "myositis," "cranial neuropathy," "mononeuropathy," "polyneuropathy," "myelopathy," "myelitis," "multiple sclerosis," "Neuromyelitis optica spectrum (NMO)," "headaches," "seizures," "psychosis," "depression," "connective tissue disorders," "rheumatoid arthritis, systemic lupus erythematosus, primary Sjögren's syndrome," "Immunological profiles," "ANA," "anti-ribosomal P antibodies," "Antiphospholipid antibodies," "SSA/SSB," "RF," "ACA," "cryoglobulins," "A-ds DNA," and "AQ4 antibody".

2.2. Inclusion and Exclusion Criteria. Articles not published in English, letters, summaries, dissertations, theses, and case reports were excluded, as well as studies that used children or animal models and studies that were not related to central and peripheral nervous complications of RA, SLE, and pSS. Studies were included only if diagnosis of neurological complications was made by expert neurologists. Only studies with neurological complications primary or secondary to RA, SLE, and PSS with associated immunological features were included. Immunological tests (i.e., gamma globulins, ANA, anti-ribosomal $\mathrm{P}$ antibodies, anti-Ro/SSA and antiLa/SSB antibodies, RF, AQ4 antibody, and cryoglobulins) were included if they were performed at the time of diagnosis and at least yearly during follow-up.

\section{Results}

The described search identified six hundred seventy-five relevant studies (Figure 1), 107 of which were excluded based on abstract analysis. The remaining 37 studies were reviewed on its full text and 22 were included in this systematic review

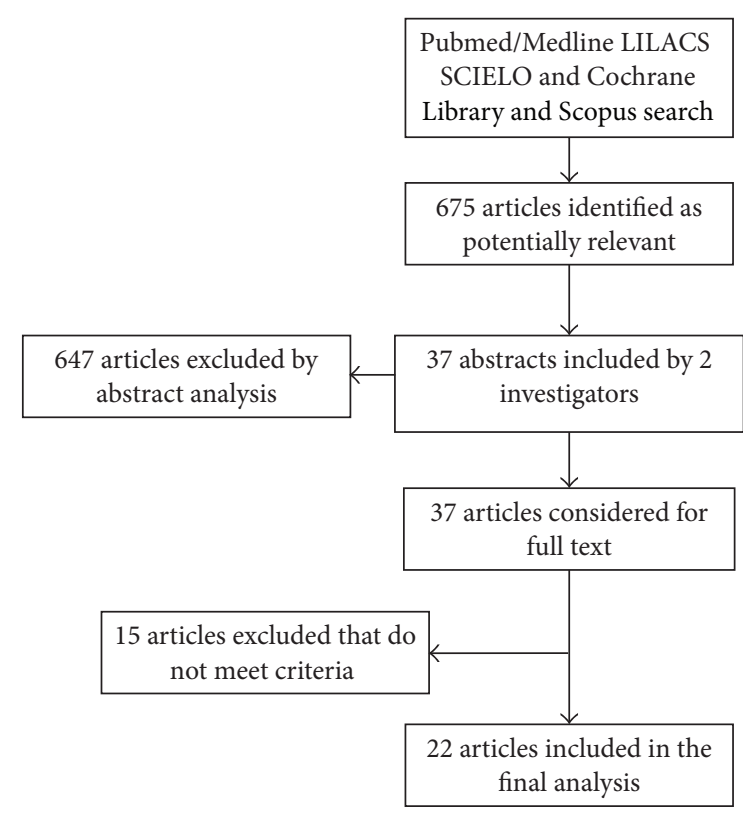

FIGURE 1: Research process flowchart.

that addressed the inclusion criteria (a total of 2338 patients). The majority of them were cohort studies (18); 1 was casecontrol and 1 was randomized controlled trial. Of these, 12 studies focused on SLE and 9 on SS. Only one study was found regarding patients with RA. Classification of each connective tissue disorders was assessed according to established criteria for SLE [6], SS [7], and RA [8].

We performed qualitative data synthesis, organizing the results by connective tissue type. We did not attempt to perform meta-analysis because of the heterogeneity of the study designs, populations, and results. Using our critical appraisal of individual studies and the body of evidence for each study design, we identified strengths and weaknesses of each study in the discussion. We did not assess publication bias. Table 2 summarizes the main methodologic characteristics and results of the included studies.

In 1989, Mellgren et al. [29] reviewed 33 cases of primary SS and peripheral neuropathy evaluated at the Mayo Clinic from 1976 to 1988 and studied sural nerve biopsy specimens in 11 of them. Based on analytical clinical and electrophysiological (electromyography and sensory and motor conduction study) nerve features, 23 patients had PNS features such as distal sensorimotor neuropathy, 10 had a sensory neuropathy, five had trigeminal neuropathy, and two had carpal tunnel syndrome. The authors did not report any correlation between neuropathy and laboratory findings such as anti-SSA/Ro antibodies, cryoglobulins, and RF in patients with SS.

In Andonopoulos et al.s study [30], 10 of the 14 patients with evidence of PNS disease had a mild peripheral neuropathy of the glove and stocking distribution; six of them were mixed and four were sensory only. Two of those, plus one more, had trigeminal neuropathy. Two patients had abnormal terminal latencies of the peroneal nerves, indicative of motor neuropathy. Six of our primary SS subjects 


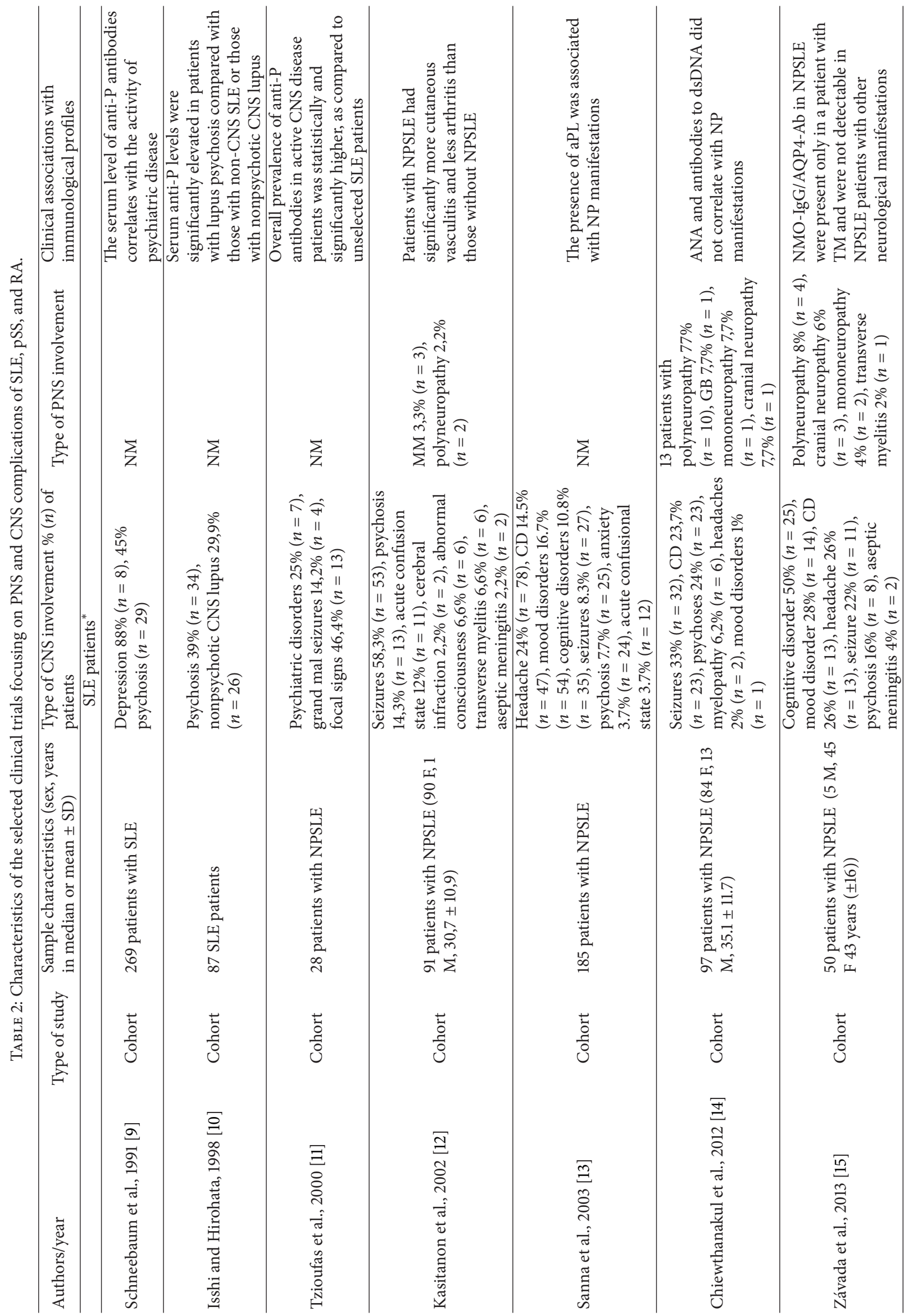




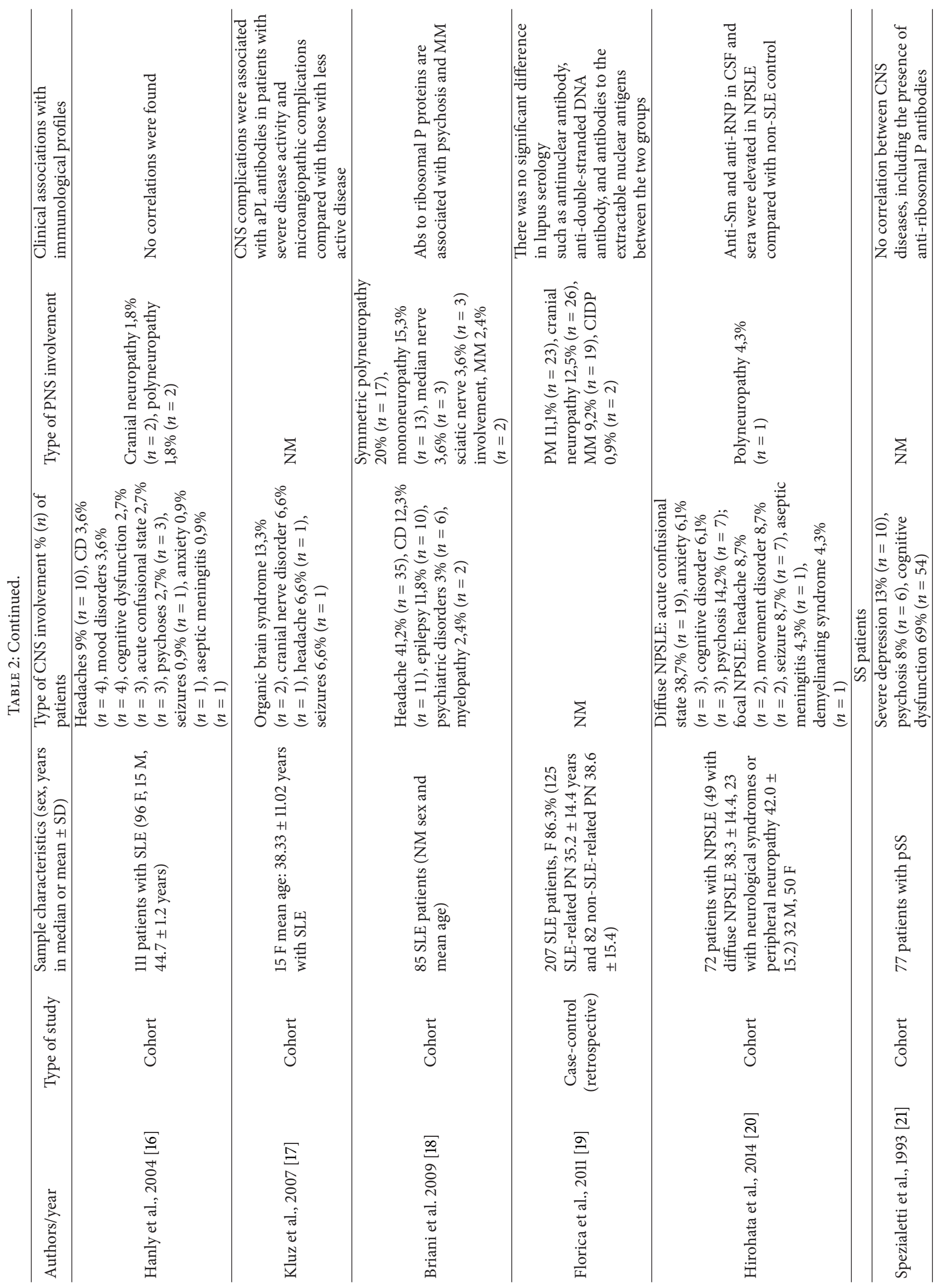




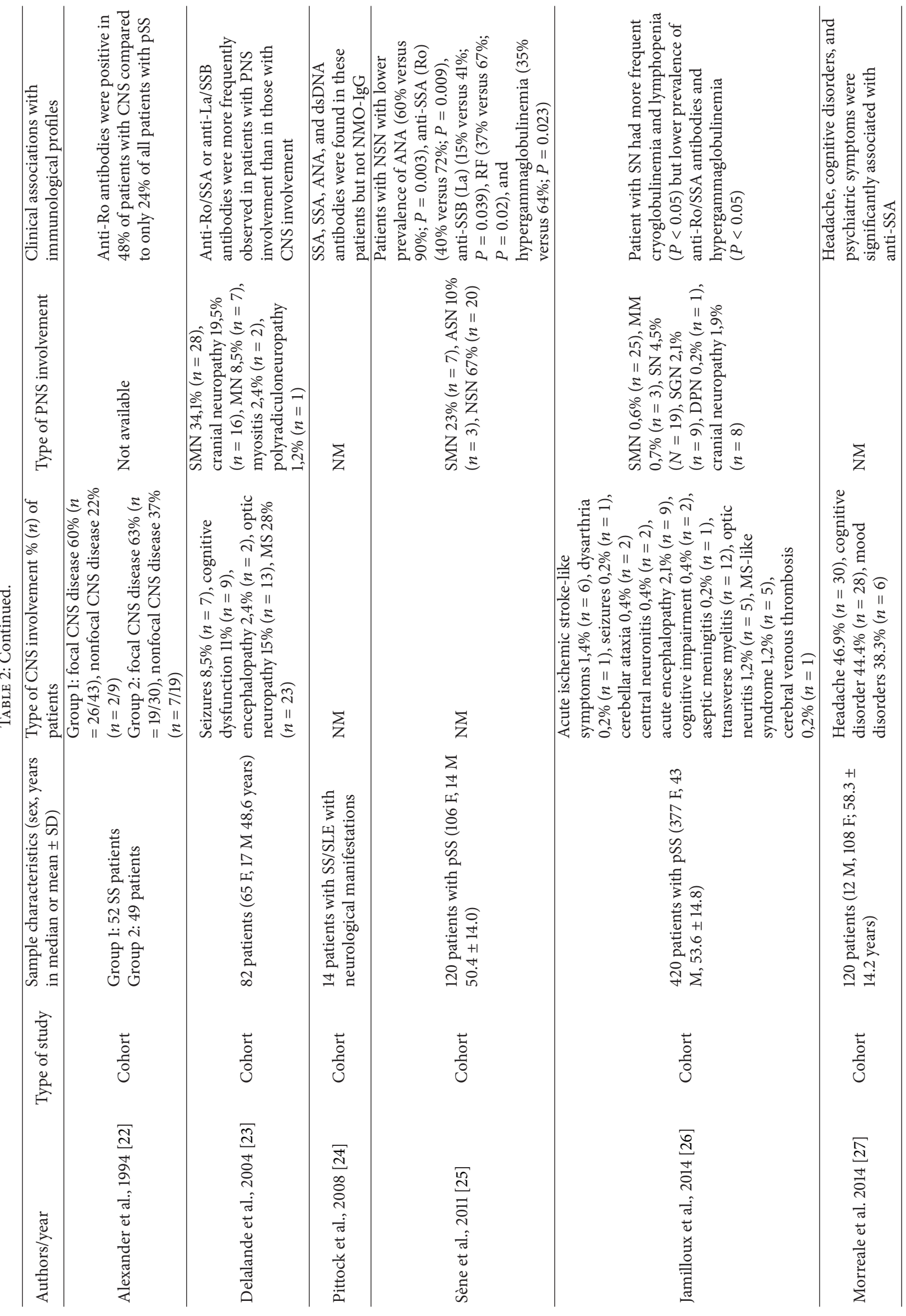




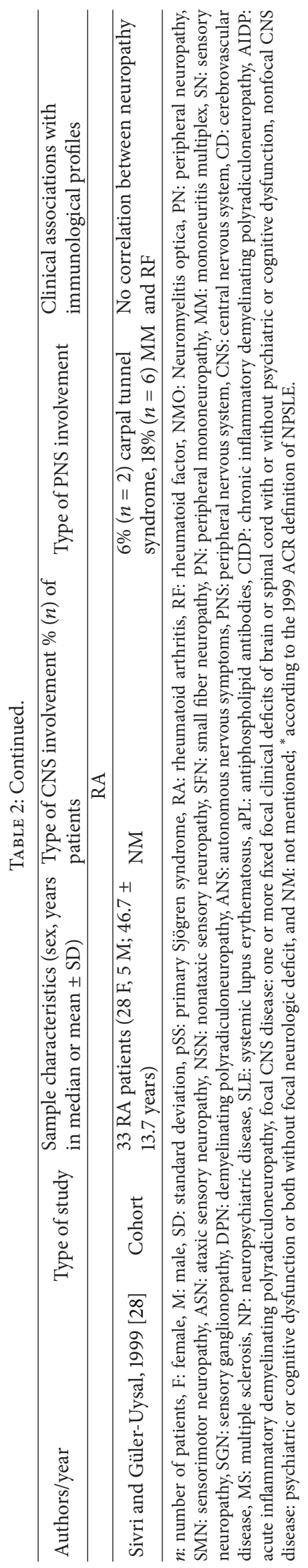


had skin vasculitis, all anti-SS-A/Ro positive. Two of those, with cryoglobulinemia and low $\mathrm{C}$, had PNS disease (one with mononeuritis multiplex). Only one patient with severe primary SS, manifested by cryoglobulinemia, vasculitis, and glomerulonephritis, presented with mononeuritis multiplex that partially responded to intravenous cyclophosphamide and high-dose steroids. Of interest is the absence of any statistically significant correlation between the observed PNS pathology and any other clinical or immunologic feature.

Among 33 RA patients, Sivri and Güler-Uysal [28] found $2(6 \%)$ patients to have carpal tunnel syndrome, while $6(18 \%)$ patients had mononeuritis multiplex. Impaired nerve conduction velocities were detected in 6 (18\%) of 33 RA patients suggesting CNS involvement with intact PNS (however, there is no information about CNS involvement). Of note, there was not any clear correlation between neuropathy and clinical or laboratory data (RF).

Kasitanon et al. [12] studied 91 patients (90 females, 1 male) fulfilling standardized criteria of ACR nomenclature for neuropsychiatric SLE (NPSLE) [31]. CNS manifestations included seizures in 53 patients (54.1\%), psychosis in 13 (13.3\%), acute confusion state in $11(11.2 \%)$, abnormal consciousness in $6(6.1 \%)$, transverse myelitis in $6(6.1 \%)$, cerebral infarction in $2(2.0 \%)$, and aseptic meningitis in $2(2.0 \%)$. PNS manifestations such as peripheral neuropathy were reported in $5(5.1 \%)$ patients. However, patients with NPSLE had significantly more cutaneous vasculitis than those without associations between NPSLE and immunoserological data.

In Schneebaum et al. study [9], 19\% of 269 patients with SLE demonstrated elevated levels of IgG or IgM anti$\mathrm{P}$ antibodies, including $14 \%$ of 187 patients without and $29 \%$ of 82 patients with neuropsychiatric manifestations. The frequency in patients with severe depression $(n=8)$ and psychosis $(n=29)$ was $88 \%$ and $45 \%$, respectively, compared with only $9 \%$ in patients with nonpsychiatric neurologic disease $(n=45)$. For the entire SLE group, the odds ratio for the association of anti-P antibodies and severe psychiatric manifestations was 7.63 with a $95 \%$ confidence interval of 3.61 to 16.14 .

Isshi and Hirohata [10] also analyzed sera from 87 SLE patients (27 with non-CNS SLE, 34 with lupus psychosis, and 26 with nonpsychotic CNS lupus) and from 20 control patients with neurologic manifestations without SLE and cerebrospinal fluid (CSF) from 41 patients with CNS lupus and from the 20 control patients for IgG anti-P. Serum anti$\mathrm{P}$ levels were significantly elevated in patients with lupus psychosis compared with those with non-CNS SLE or those with nonpsychotic CNS lupus, indicating that anti-P in the systemic circulation are involved in the development of lupus psychosis.

In Tzioufas et al. study [11], serum was obtained from 28 SLE patients during active CNS involvement. Eleven patients had diffuse CNS involvement (seven had psychiatric disorders and four had grand mal seizures), 13 patients had focal CNS involvement, seven of whom were associated with antibodies to cardiolipin, and four patients had a mixed form of CNS disease. The overall prevalence of anti-P antibodies in active CNS disease patients was statistically and significantly higher, as compared to unselected SLE patients $\left(\chi^{2}=6.04\right.$, $P<0.05)$. These antibodies are associated with active SLE and CNS involvement particularly in patients without anticardiolipin antibodies.

In Sanna et al. study [13], 185 SLE patients (57.3\%) had NP manifestations at any time during follow-up. Headache was the most frequent manifestation, present in 78 patients $(24 \%)$. Cerebrovascular disease (CD) was diagnosed in $47 / 323$ patients (14.5\%), with a total of 57 events. Mood disorders were found in 54 (16.7\%), cognitive disorders in 35 (10.8\%), and seizures in 27 patients $(8.3 \%)$. Psychosis was diagnosed in $25(7.7 \%)$, anxiety disorder in 24 (3.7\%), and acute confusional state in 12 patients (3.7\%). NP manifestations are significantly associated with aPL. CD, headache, and seizures were independently associated with these antibodies.

In Hirohata et al. study [20], 23 patients showed that CNS manifestations other than diffuse NPSLE, including neurologic syndromes and PNS involvements (focal NPSLE), according to the ACR nomenclature for NPSLE anti-Sm levels in CSF and sera were measured using enzyme-linked immunosorbent assay (ELISA) kits. Anti-Sm in CSF were significantly elevated in NPSLE compared with non-SLE control. Among subsets of NPSLE, CSF anti-Sm was significantly elevated in acute confusional state compared with nonacute confusional state diffuse NPSLE $(P=0.0028)$ or with focal NPSLE $(P=0.0008)$.

In Závada et al. study [15], sera of 76 patients with SLE and neurological symptoms, 50 of whom met the ACR nomenclature for NPSLE, were tested for AQP4-Ab in an indirect immunofluorescence assay employing HEK293 cells transfected with recombinant human AQP4. Only one of the examined sera was positive for NMO-IgG/AQP4-Ab. None of the 75 NPSLE without was found to be seropositive for NMO-IgG/AQP4-Ab. NMO-IgG/AQP4-Ab in NPSLE were present only in a patient with transverse myelitis and were not detectable in NPSLE patients with other neurological manifestations.

Hanly et al. [16] identified 41 NPSLE patients. CNS manifestations accounted for $92 \%$ of the events compared to involvement of the PNS in $8 \%$. In patients with NP, the occurrence of renal disease was significantly associated with use of prednisone and immunosuppressive drugs. However, associations between positive antinuclear antibodies (97\%) and elevated anti-dsDNA antibodies were not examined.

According to 1997 American College of Rheumatology diagnostic criteria [6], 15 women were enrolled in the study of Kluz et al. [17] with severe disease activity (a modified SLE Disease Activity Index (SLEDAI) of $>12$ points) and inflammatory microangiopathy-related complications such as systemic CNS affection and/or vasculitis and/or nephritis. In 6 of them, CNS complications were seizure $(n=1)$, organic brain syndrome $(n=2)$, cranial nerve disorder $(n=1)$, and headache $(n=2)$. Significantly higher levels of both fractions of ACA and IgM ACA and of both fractions of ACA and IgM ACA and antiphospholipid antibodies (repeatedly positive test for serum ACA and/or lupus anticoagulant) were determined in patients with severe disease activity and microangiopathic complications compared with those with less active disease. 
Briani et al. [18] presented 85 SLE patients (39\%) with neurological manifestations. Both central (53 patients) and peripheral (23 patients) nervous systems were involved (Table 2). In 9 patients CNS and PNS manifestations concurred. The most common CNS manifestation was headache (35 cases, 58\%) followed by cerebrovascular diseases (11 cases, $17 \%$ ), epilepsy (10 cases, $16 \%$ ), psychiatric disorders (6 cases, $10 \%$ ), and myelopathy (2 cases, 3\%). PNS manifestations included symmetric polyneuropathy (17 cases, 20\%), mononeuropathy (13 cases, 10 with median nerve and 3 with sciatic nerve involvement, 35\%), and mononeuropathy multiplex ( 2 cases, $5 \%$ ). One patient with mononeuropathy multiplex underwent sural nerve biopsy, which showed vasculitic axonal injury. Of the 7 sera and CSF samples with Abs to ribosomal $\mathrm{P}$ proteins, these autoantibodies are associated with CNS manifestations such as psychosis $(P=0.017)$ and PNS mononeuropathy multiplex $(P=0.040)$.

Gono et al. [32] enrolled 20 patients with pSS. PNS involvement regarded seven patients with cranial neuropathy, nine patients with polyneuropathy, and three patients with mononeuritis multiplex (Table 2). Seven patients with cranial neuropathy included three with optic neuritis, two with trigeminal neuralgia, one with facial nerve palsy, and one with glossopharyngeal and vagus nerve palsy. Eight of the nine patients with polyneuropathy revealed pure sensory neuropathy. Two of the three patients with mono neuritis multiplex revealed motor sensory neuropathy. CNS involvement included three patients with encephalopathy and two patients with aseptic meningitis. One patient with optic neuritis had spinal cord lesions and cerebral focal symptoms, such as right hemiparesis, which are similar findings in multiple sclerosis. Moreover, AQ4 antibody was detected in the other one of the three patients with optic neuritis. This patient was diagnosed with Neuromyelitis optica (NMO) associated with pSS.

Among 207 SLE patients Florica et al. [19] showed that 125 had SLE-related PN involvement and 82 had non-SLE-related PN. The most frequent etiologies of non-SLE-related PN were compressive neuropathy (nerve or root compression) in $35(42.6 \%)$, followed by medication toxicity in $23(28 \%)$, hypothyroidism in $19(23.1 \%)$, and diabetes mellitus in 14 (17\%). Other causes of non-SLE-related PN included ethanol abuse, paraproteinemia, uremia, and viral hepatitis in a limited number of patients.

Patients with PNS were more likely to have also CNS involvement (14.2\%) compared with patients without PN (CNS involvement: $6.6 \%, P=0.02$ ). The most common PN observed was peripheral polyneuropathy, with a predominance of the sensory form, present in 76 (36.7\%) patients and the sensory-motor variant in $39(18.8 \%)$ patients. Twentythree $(11.1 \%)$ patients had a peripheral mononeuropathy and $26(12.5 \%)$ had a cranial neuropathy. Nineteen $(9.2 \%)$ patients suffered from mononeuritis multiplex and only 11 (5.3\%) were diagnosed with chronic inflammatory demyelinating polyradiculoneuropathy (CIDP). There was no difference between PNS features and SLE immune markers (presence of antiphospholipid antibodies).

In study of Chiewthanakul et al. [14], CNS manifestations accounted for $87 \%$ (84/97 patients), while involvement of the
PNS was $13 \%$ (13/97 patients). The three most frequent CNS manifestations included seizures (33\%), psychoses $(22.7 \%)$, and cerebrovascular disease $(22.3 \%)$, composed of $82.6 \%$ cerebral infarction, $8.7 \%$ transient ischemic attack, and $8.7 \%$ venous sinus thrombosis. ANA and antibodies to dsDNA did not correlate with NP manifestations.

In 2014 study by Jamilloux et al. [26], 420 patients fulfilled the 2002 American-European pSS criteria [7]. Within 93 (22\%) patients with neurological manifestations, PN and CNS were involved in $66 \%$ and $44 \%$, respectively. The number of extraglandular manifestations was increased in patients with sensorimotor neuropathies $(3.3 \pm 1.9$ versus $2 \pm 1.5, P<$ $0.05)$. This subgroup had more frequent cryoglobulinemia and lymphopenia $(P<0.05)$ but lower prevalence of anti-Ro/SSA antibodies and hypergammaglobulinemia $(P<$ 0.05). Authors also confirmed cryoglobulinemia as unique predictive factor of PNS disease.

In Spezialetti et al. study [21], from 77 patients with pSS and CNS involvement, psychiatric or cognitive impairment, usually mild or moderate, occurred in over $80 \%$ (63 of 77) of this highly selected population of SS patients, and more than $60 \%$ of patients (48 of 77 ) had both. Anti-ribosomal $\mathrm{P}$ antibodies occurred in six (4.6\%) patients with SS and related disorders. None of the patients with primary SS had anti-ribosomal $\mathrm{P}$ antibodies. There was no correlation between nonfocal CNS disease, including psychosis or severe depression, and the presence of anti-ribosomal $\mathrm{P}$ antibodies. Paired serum CSF samples from 34 SS patients with active CNS disease, including 6 with psychosis and 5 with severe depression, did not contain anti-ribosomal P.

In Sène et al. study [25], patients with pSS and PNS involvement and nonataxic sensory neuropathy were characterized by a higher age $(57.5 \pm 10.7$ versus $48.7 \pm 14.3$ years; $P=0.007)$, more frequent CNS involvement (15\% versus $2 \% ; P=0.04)$, and lower prevalence of ANA (60\% versus 90\%; $P=0.003)$, anti-SSA (Ro) $(40 \%$ versus $72 \% ; P=$ 0.009 ), anti-SSB (La) (15\% versus $41 \%$; $P=0.039)$, RF $(37 \%$ versus $67 \% ; P=0.02)$, and hypergammaglobulinemia $(35 \%$ versus $64 \% ; P=0.023)$. In multivariate analysis, nonataxic sensory neuropathy was associated with the presence of CNS involvement (OR, 17.0; $P=0.025$ ) and ANA (OR, 0.07; $P<$ 0.001).

In Delalande et al. study [23], fifty-six patients with pSS had CNS disorders, which were mostly focal or multifocal. Twenty-nine patients had spinal cord involvement (acute myelopathy $(n=12)$, chronic myelopathy $(n=16)$, or motor neuron disease $(n=1))$. The disease mimicked relapsing-remitting multiple sclerosis (MS) in 10 patients and primary progressive MS in 13 patients. CNS symptoms included seizures $(n=7)$, cognitive dysfunction $(n=9)$, and encephalopathy $(n=2)$. Fifty-one patients had PNS involvement: symmetric axonal sensorimotor polyneuropathy with a predominance of sensory symptoms or pure sensory neuropathy occurred most frequently $(n=28)$, followed by cranial nerve involvement affecting trigeminal, facial, or cochlear nerves $(n=16)$. Multiple mononeuropathy $(n=$ $7)$, myositis $(n=2)$, and polyradiculoneuropathy $(n=1)$ were also observed. Anti-Ro/SSA or anti-La/SSB antibodies were detected in $21 \%$ of patients at the diagnosis of SS, more 
frequently observed in patients with PNS involvement than in those with CNS involvement $(P<0.01)$.

Alexander et al. [22] examined 49 SS patients (group I) for the potential relationship between anti-Ro(SS-A) antibodies and active CNS disease by double immunodiffusion. In group 2 with 52 SS patients and active CNS disease, the anti-SSA antibodies were detected by ELISA. This study showed that anti-Ro antibodies were positive in $48 \%$ of patients with CNS manifestations and serious complications compared to only $24 \%$ of all patients with pSS.

Pittock et al. [24] detected NMO-IgG in 5 of 14 patients (35.7\%) with NMOSDs and SS/SLE and in 2 of 4 patients (50.0\%) with NMO without SS/SLE $(P=0.59)$. SSA, SSA, ANA, and dsDNA antibodies were found in these patients but not NMO-IgG.

\section{Discussion}

In this first systematic review of both CNS and PNS involvement in $R A, S L E$, and $p S S$, our results indicate that CNS complications (9-92\%) are much more frequent than those of PNS (8-66\%) across the various studies (total of 2338 patients). These findings are compatible with previous results in the literature. In this review, the majority of reported studies attributable in SLE CNS manifestations range from 13 to $92 \%$ of the events compared to PNS in $8-56 \%$. The most frequent NP syndromes were headache, mood disorders, and seizures which are consistent with previous meta-analysis [33]. Ours finding are also consistent with previous studies where SLE CNS manifestations range from 33\% to $75 \%$ [34, 35]. In SS studies, CNS manifestations range between 9 and $44 \%$ and PNS ranged between 24 and 68\%, which agree with previous studies [36]. In this review, the frequency of PNS (mononeuritis) in RA patients is $18 \%$ lower as compared to other study, while for entrapment neuropathy $(6 \%$ in our patients) it varies from $4 \%$ to $54.6 \%[37,38]$.

Overall prevalence is seen to vary widely among the studies and is mainly attributed to a number of factors including bias in selection of patients for study, disease duration, and lack of uniformity in diagnostic criteria. With this in mind, we sought to expand the probability of specific manifestations by using established criteria and doublereading (by a certified neurologist), and excluding uncertain cases.

Among the most interesting points of this review is the correlation between CNS/PNS manifestations and immune activation markers (i.e., antinuclear antibodies ANA, antiRo/SSA, anti-La/SSB, RF, and hypergammaglobulinemia). Anti-Ro and anti-La seem to be less frequent in pSS patients with neurological involvement (40\%) compared to patients without neurological manifestations (60\% of positivity), so, it is necessary for more markers are necessary in pSS to better classify subpopulations of patients with neurological involvement [39]. Jamilloux et al. [26] claimed that the implication that the immunological profile (as potential serological markers) may only be a signature related to distinct neurological rather than a reflection of the pathogenic mechanism could not be dismissed. Nonetheless, given the apparent association with Raynaud's phenomenon, cutaneous vasculitis, and renal involvement, they proposed that sensorimotor neuropathy or mononeuritis multiplex originates from immunovascular injury. Contrary to other studies, Jamilloux et al. showed that sensory ganglionopathy is associated with lymphocytic infiltration and not cryoglobulinemia. In the same manner, Mellgren et al. showed that sensory ganglionopathy in pSS was related to lymphocyte infiltration of the dorsal root ganglia. In addition, Gono et al. reported optic neuropathy in one patient with SS and NMO (Aquaporin Ab positive) and MS, suggesting the implication of more mechanisms associated with the pathogenesis of neurological involvement in SS rather than the previously described vasculopathy. In the same context, Pittock et al. concluded that NMO disorders with seropositive findings for NMO-IgG occurring with SS/SLE or non-organ-specific autoantibodies is an indication of coexisting NMO rather than a vasculopathic or other complication of SS/SLE. Florica et al. observed that the group of SLE-related PN patients had more frequent CNS involvement and a higher score of disease activity, indicating that the immune response shows a preference toward neurological tissue. This is the first large study solely focused on peripheral neuropathy in SLE which suggests an autoimmune etiology for PN manifestations in SLE. It remains undetermined whether any of these clinical characteristics could predict the onset of PNS or/and CNS in either SS or SLE or other connective tissue disorders.

Several specific autoantibodies are associated with NPSLE (antiphospholipid, serum anti-ribosomal P antibodies, ANA, and antibodies to dsDNA). The presence of antiphospholipid antibodies is significantly associated with cerebrovascular disease and cognitive dysfunction, whereas serum antiribosomal $\mathrm{P}$ antibodies are strongly associated with psychosis and depression in SLE as discussed in this review $[9,10,13$, 20]. These findings are consistent with one multicenter study [40]. Moreover, the presence of cutaneous vasculitic lesions was significantly associated with NPSLE in the study by Kasitanon et al., rather than thrombocytopenia, as suggested by other studies [41]. According to Chiewthanakul et al., cutaneous vasculitis was only marginally related to active CNS involvement. Notably, in this study, neither ANA and antibodies to dsDNA nor anti-ribosomal P antibodies correlated with NP manifestations. In the same line is Spezialetti et al. study [21]. It seems that the independent associations of such manifestations with NPSLE have been difficult to study so far. This is partly explained by the difficulty to conduct studies with an adequate sample size.

However, Andonopoulos et al. failed to find a statistically significant correlation between the observed PNS pathology and any other clinical or immuno/serologic data. In the absence of any obvious reason, it is tempting to ascribe PNS symptoms to vasculitis of the vasa nervorum, but the data provided cannot fully support this hypothesis. The classical severe picture of a patient presenting with mononeuritis multiplex, associated with vasculitis in other organs, as already mentioned, leaves little doubt as to the pathogenesis of the neurologic syndrome. However, no patient has been reported to exhibit additional features of CNS disease that could be clinically detected. 
Similarly, Sivri and Güler-Uysal found no correlation between mononeuritis and the immunological data in the serum of RA patients (i.e., RF), which is in contrast with the finding by other study [42]. This could be explained by the implication of a number of multiple factors in determining the clinical signs of disease (i.e., nonsteroidal antiinflammatory drugs, NSAIDs). The single center, tertiary referral center-based patient population and small number of subjects might also explain the result.

Several limitations which compromised their external validity were identified in the specific studies, mainly by their authors. In terms of reporting complications, methods varied considerably. In this review, incidence was determined from studies of varying sample sizes across multiple age groups. Data collection was retrospective $[14,19,29,32]$. Hence, patient assessment might have been incomplete, rendering it difficult to analyze details of NPSLE syndromes [13] or missing PN initial events [20]; secondly, other CNS manifestations, such as headaches, anxiety, cognitive dysfunction, and autonomic disorders, may have been missed; thus, their prevalence has been underestimated $[19,30]$. Finally, the cause of death by autopsy was not always performed; thus, authors could not conclude whether death was related to SLE-mediated organ dysfunction or other diseases [15]. Perhaps due to methodological limitations it was not possible to determine if the associations between medication use with the occurrence of NP events and renal disease were independent of each other [9].

Notwithstanding, this review is not without its limitations. There are a small number of studies available for analysis. One limitation of this review is that a limited number of databases were searched. Consequently, there is always the possibility that we overlooked studies in other databases published before 1980. In addition, there is always the possibility of publication bias due to underreported negative results and grey literature. However, the use of fewer search limits increases the sensitivity of the search method.

\section{Conclusions}

Despite the abovementioned limitations, this systematic review provides foundation for further research on the pathophysiology of immune system among the vasculature of the CNS and PNS. It is still a matter of debate whether the CNS and PNS manifestations of RA, SLE, and pSS are direct effect of these diseases or secondary such as side effects of corticosteroids and other immunosuppressive therapies or multiple systemic organ dysfunction (i.e., drug or SLE-mediated multiorgan induced psychosis). To date, no laboratory test, that is, autoantibodies, has been shown as pathognomonic neither for CNS nor for PNS involvement in these connective tissue disorders. Prospective multicenter studies are warrant for further confirmations.

\section{Conflict of Interests}

The authors declare that there is no conflict of interests regarding the publication of this paper.

\section{References}

[1] J. E. Riggs, "Neurological consequences of systemic disease," in Neurology in Clinical Practice, W. Bradley, R. Daroff, G. Fenichel, and D. Marsden, Eds., pp. 841-860, ButterworthHeinemann, Boston, Mass, USA, 1991.

[2] C. Chalk, J. Dyck, and D. Conn, "Vasculitic neuropathy," in Peripheral Neuropathy, P. K. Thomas and P. J. Dyck, Eds., pp. 1424-1430, Saunders, Philadelphia, Pa, USA, 1993.

[3] G. Midroni and J. Bilbao, "Vasculitic neuropathy," in Biopsy Diagnosis of Peripheral Neuropathy, G. Midroni and J. Bilbao, Eds., pp. 241-262, Butterworth-Heinemann, Boston, Mass, USA, 1995.

[4] E. V. Voss and M. Stangel, "Nervous system involvement of connective tissue disease: mechanisms and diagnostic approach," Current Opinion in Neurology, vol. 25, no. 3, pp. 306-315, 2012.

[5] S. Sciascia, M. L. Bertolaccini, D. Roccatello, M. A. Khamashta, and G. Sanna, "Autoantibodies involved in neuropsychiatric manifestations associated with systemic lupus erythematosus: a systematic review," Journal of Neurology, vol. 261, no. 9, pp. 1706-1714, 2014.

[6] M. C. Hochberg, "Updating the American College of Rheumatology revised criteria for the classification of systemic lupus erythematosus [letter]," Arthritis \& Rheumatism, vol. 40, no. 9, p. $1725,1997$.

[7] C. Vitali, S. Bombardieri, R. Jonsson et al., "Classification criteria for Sjögren's syndrome: a revised version of the European criteria proposed by the American-European Consensus Group," Annals of the Rheumatic Diseases, vol. 61, no. 6, pp. 554$558,2002$.

[8] D. Aletaha, T. Neogi, A. J. Silman et al., "2010 rheumatoid arthritis classification criteria: an American College of Rheumatology/European League Against Rheumatism collaborative initiative," Annals of the Rheumatic Diseases, vol. 69, no. 9, pp. 1580-1588, 2010.

[9] A. B. Schneebaum, J. D. Singleton, S. G. West et al., "Association of psychiatric manifestations with antibodies to ribosomal p proteins in systemic lupus erythematosus," The American Journal of Medicine, vol. 90, no. 1, pp. 54-62, 1991.

[10] K. Isshi and S. Hirohata, "Differential roles of the anti-ribosomal $\mathrm{P}$ antibody and antineuronal antibody in the pathogenesis of central nervous system involvement in systemic lupus erythematosus," Arthritis and Rheumatism, vol. 41, no. 10, pp. 18191827, 1998.

[11] A. G. Tzioufas, N. G. Tzortzakis, E. Panou-Pomonis et al., "The clinical relevance of antibodies to ribosomal-P common epitope in two targeted systemic lupus erythematosus populations: a large cohort of consecutive patients and patients with active central nervous system disease," Annals of the Rheumatic Diseases, vol. 59, no. 2, pp. 99-104, 2000.

[12] N. Kasitanon, W. Louthrenoo, S. Piyasirisilp, W. Sukitawut, and R. Wichainun, "Neuropsychiatric manifestations in Thai patients with systemic lupus erythematosus," Asian Pacific Journal of Allergy and Immunology, vol. 20, no. 3, pp. 179-185, 2002.

[13] G. Sanna, M. L. Bertolaccini, M. J. Cuadrado et al., "Neuropsychiatric manifestations in systemic lupus erythematosus: prevalence and association with antiphospholipid antibodies," Journal of Rheumatology, vol. 30, no. 5, pp. 985-992, 2003. 
[14] P. Chiewthanakul, K. Sawanyawisuth, C. Foocharoen, and S. Tiamkao, "Clinical features and predictive factors in neuropsychiatric lupus," Asian Pacific Journal of Allergy and Immunology, vol. 30, no. 1, pp. 55-60, 2012.

[15] J. Závada, P. Nytrová, K. P. Wandinger et al., "Seroprevalence and specificity of NMO-IgG (anti-aquaporin 4 antibodies) in patients with neuropsychiatric systemic lupus erythematosus," Rheumatology International, vol. 33, no. 1, pp. 259-263, 2013.

[16] J. G. Hanly, G. McCurdy, L. Fougere, J.-A. Douglas, and K. Thompson, "Neuropsychiatric events in systemic lupus erythematosus: attribution and clinical significance," The Journal of Rheumatology, vol. 31, no. 11, pp. 2156-2162, 2004.

[17] J. Kluz, W. Kopeć, U. Jakobsche, I. Prajs, and R. Adamiec, "Vasculitis in systemic lupus erythematosus (SLE) - assessment of peripheral blood mononuclear cell activation and the degree of endothelial dysfunction: initial report," Postpy Higieny I Medycyny Doświadczalnej, vol. 61, pp. 725-735, 2007.

[18] C. Briani, M. Lucchetta, A. Ghirardello et al., "Neurolupus is associated with anti-ribosomal $\mathrm{P}$ protein antibodies: an inception cohort study," Journal of Autoimmunity, vol. 32, no. 2, pp. 79-84, 2009.

[19] B. Florica, E. Aghdassi, J. Su, D. D. Gladman, M. B. Urowitz, and P. R. Fortin, "Peripheral neuropathy in patients with systemic lupus erythematosus," Seminars in Arthritis and Rheumatism, vol. 41, no. 2, pp. 203-211, 2011.

[20] S. Hirohata, Y. Sakuma, T. Yanagida, and T. Yoshio, "Association of cerebrospinal fluid anti-Sm antibodies with acute confusional state in systemic lupus erythematosus," Arthritis Research \& Therapy, vol. 16, no. 5, article 450, 2014.

[21] R. Spezialetti, H. G. Bluestein, J. B. Peter, and E. Alexander, "Neuropsychiatric disease in Sjögren's syndrome: antiribosomal P and anti-neuronal antibodies," American Journal of Medicine, vol. 95, no. 2, pp. 153-160, 1993.

[22] E. L. Alexander, M. R. Ranzenbach, A. J. Kumar et al., "Anti-Ro(SS-A) autoantibodies in central nervous system disease associated with Sjögren's syndrome (CNS-SS)," Neurology, vol. 44, no. 5, pp. 899-908, 1994.

[23] S. Delalande, J. de Seze, A.-L. Fauchais et al., "Neurologic manifestations in primary Sjögren syndrome: a study of 82 patients," Medicine, vol. 83, no. 5, pp. 280-291, 2004.

[24] S. J. Pittock, V. A. Lennon, J. de Seze et al., "Neuromyelitis optica and non-organ-specific autoimmunity," Archives of Neurology, vol. 65 , no. 1 , pp. 78-83, 2008.

[25] D. Sène, M. Jallouli, J.-P. Lefaucheur et al., "Peripheral neuropathies associated with primary sjögren syndrome: immunologic profiles of nonataxic sensory neuropathy and sensorimotor neuropathy," Medicine, vol. 90, no. 2, pp. 133-138, 2011.

[26] Y. Jamilloux, L. Magy, J.-F. Hurtevent et al., "Immunological profiles determine neurological involvement in Sjögren's syndrome," European Journal of Internal Medicine, vol. 25, no. 2, pp. 177-181, 2014.

[27] M. Morreale, P. Marchione, P. Ciacomini et al., "Neurological involvement in primary Sjögren syndrome: a focus on central nervous system," PLoS ONE, vol. 9, no. 1, Article ID e84605, 2014.

[28] A. Sivri and F. Güler-Uysal, "The electroneurophysiological findings in rheumatoid arthritis patients," Electromyography and Clinical Neurophysiology, vol. 39, no. 7, pp. 387-391, 1999.

[29] S. I. Mellgren, D. L. Conn, J. C. Stevens, and P. J. Dyck, "Peripheral neuropathy in primary Sjogren's syndrome," Neurology, vol. 39, no. 3, pp. 390-394, 1989.
[30] A. P. Andonopoulos, G. Lagos, A. A. Drosos, and H. M. Moutsopoulos, "Neurologic involvement in primary Sjögren's syndrome: a preliminary report," Journal of Autoimmunity, vol. 2, no. 4, pp. 485-488, 1989.

[31] "The American College of Rheumatology nomenclature and case definitions for neuropsychiatric lupus syndromes," Arthritis \& Rheumatism, vol. 42, no. 4, pp. 599-608, 1999.

[32] T. Gono, Y. Kawaguchi, Y. Katsumata et al., "Clinical manifestations of neurological involvement in primary Sjögren's syndrome," Clinical Rheumatology, vol. 30, no. 4, pp. 485-490, 2011.

[33] A. Unterman, J. E. S. Nolte, M. Boaz, M. Abady, Y. Shoenfeld, and G. Zandman-Goddard, "Neuropsychiatric syndromes in systemic lupus erythematosus: a meta-analysis," Seminars in Arthritis and Rheumatism, vol. 41, no. 1, pp. 1-11, 2011.

[34] G. A. W. Bruyn, "Controversies in lupus: nervous system involvement," Annals of the Rheumatic Diseases, vol. 54, no. 3, pp. 159-167, 1995.

[35] R. L. Brey, S. L. Holliday, A. R. Saklad et al., "Neuropsychiatric syndromes in lupus: prevalence using standardized definitions," Neurology, vol. 58, no. 8, pp. 1214-1220, 2002.

[36] J. Font, M. Ramos-Casals, G. De la Red et al., "Pure sensory neuropathy in primary Sjögren's syndrome. Longterm prospective followup and review of the literature," Journal of Rheumatology, vol. 30, no. 7, pp. 1552-1557, 2003.

[37] V. Agarwal, R. Singh, Wiclaf et al., "A clinical, electrophysiological, and pathological study of neuropathy in rheumatoid arthritis," Clinical Rheumatology, vol. 27, no. 7, pp. 841-844, 2008.

[38] S. A. Hamed, E. A. Hamed, A. M. Elattar, M. S. A. Rahman, and N. F. Amine, "Cranial and peripheral neuropathy in rheumatoid arthritis with special emphasis to II, V, VII and XI cranial nerves," APLAR Journal of Rheumatology, vol. 9, no. 3, pp. 216226, 2006.

[39] G. J. Tobn, J.-O. Pers, V. Devauchelle-Pensec, and P. Youinou, "Neurological disorders in primary Sjögren's syndrome," Autoimmune Diseases, vol. 2012, Article ID 645967, 11 pages, 2012.

[40] M. Mahler, K. Kessenbrock, M. Szmyrka et al., "International multi-center evaluation of autoantibodies to ribosomal $\mathrm{P}$ proteins," Clinical and Vaccine Immunology, vol. 13, no. 1, pp. 77-83, 2006.

[41] F. B. Karassa, J. P. A. Ioannidis, G. Touloumi, K. A. Boki, and H. M. Moutsopoulos, "Risk factors for central nervous system involvement in systemic lupus erythematosus," $Q J M$, vol. 93, no. 3, pp. 169-174, 2000.

[42] M. Biswas, A. Chatterjee, S. K. Ghosh, S. Dasgupta, K. Ghosh, and P. K. Ganguly, "Prevalence, types, clinical associations, and determinants of peripheral neuropathy in rheumatoid patients," Annals of Indian Academy of Neurology, vol. 14, no. 3, pp. 194197, 2011. 


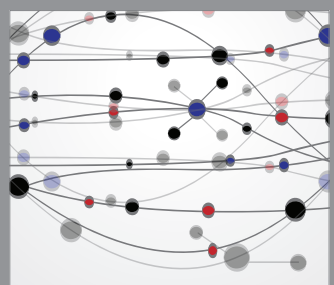

The Scientific World Journal
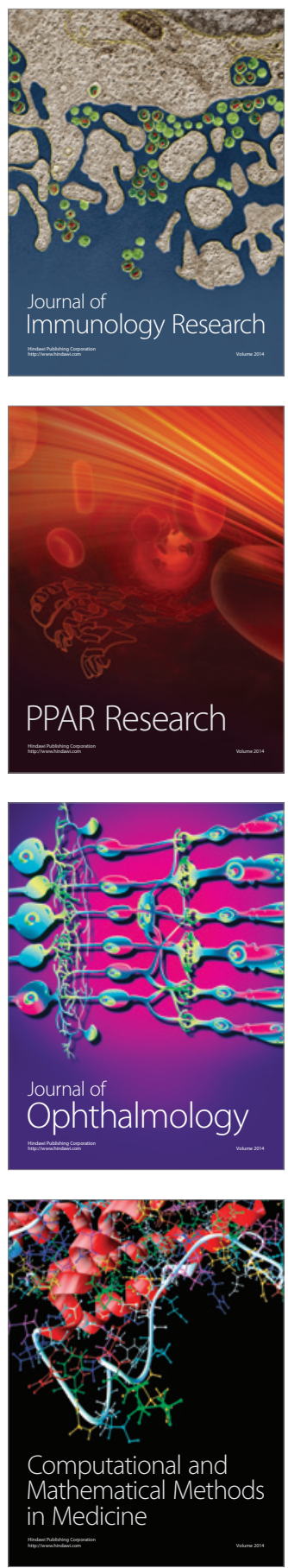

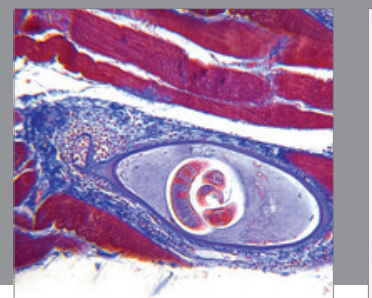

Gastroenterology

Research and Practice
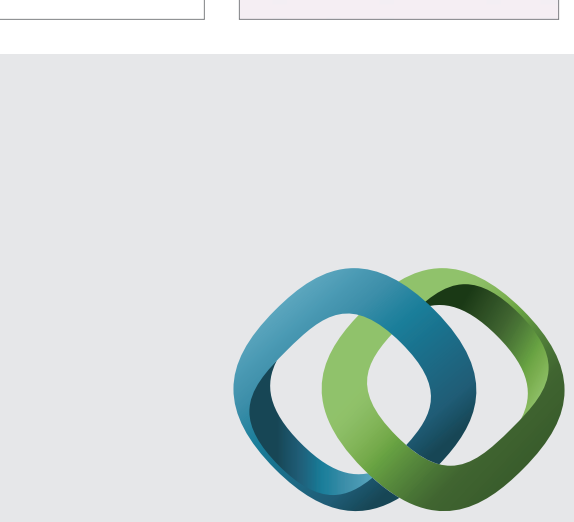

\section{Hindawi}

Submit your manuscripts at

http://www.hindawi.com
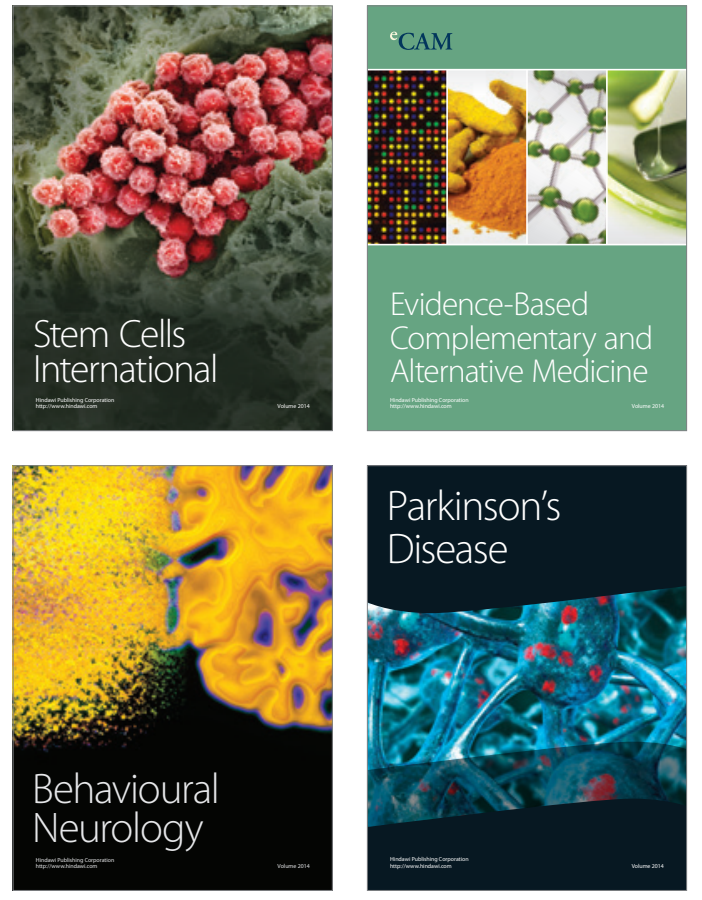
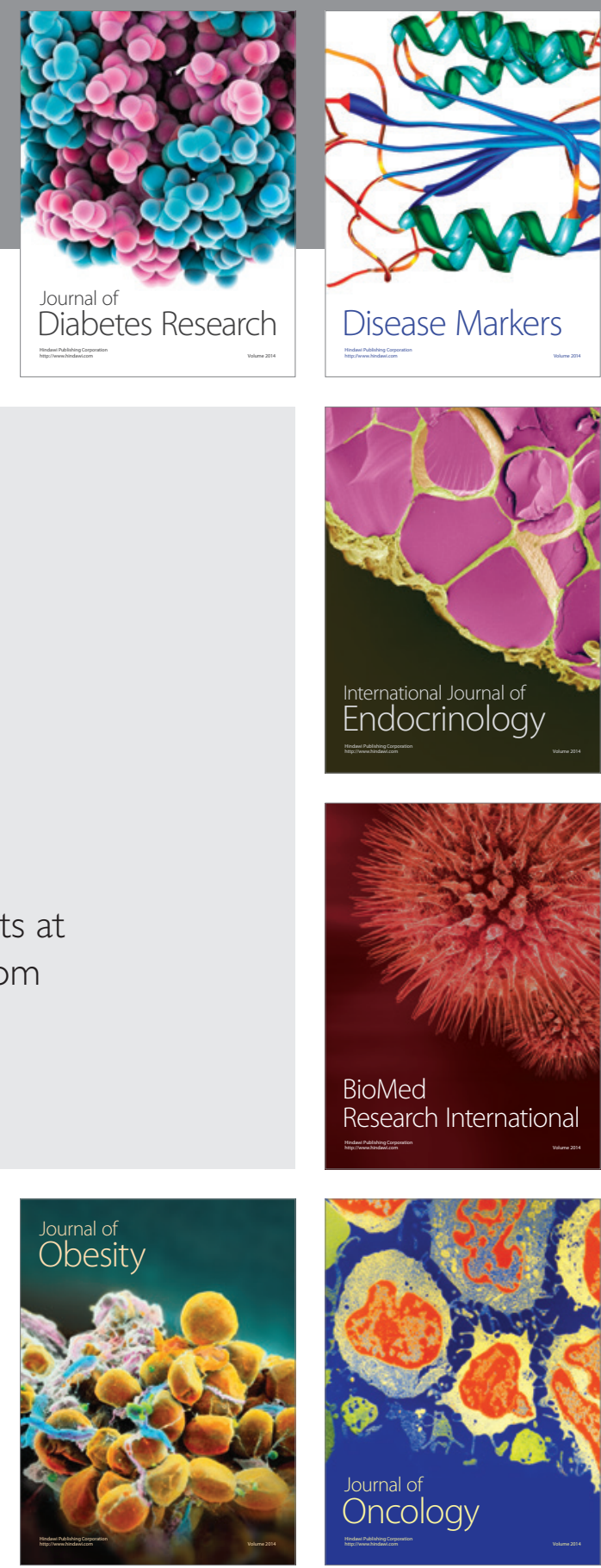

Disease Markers
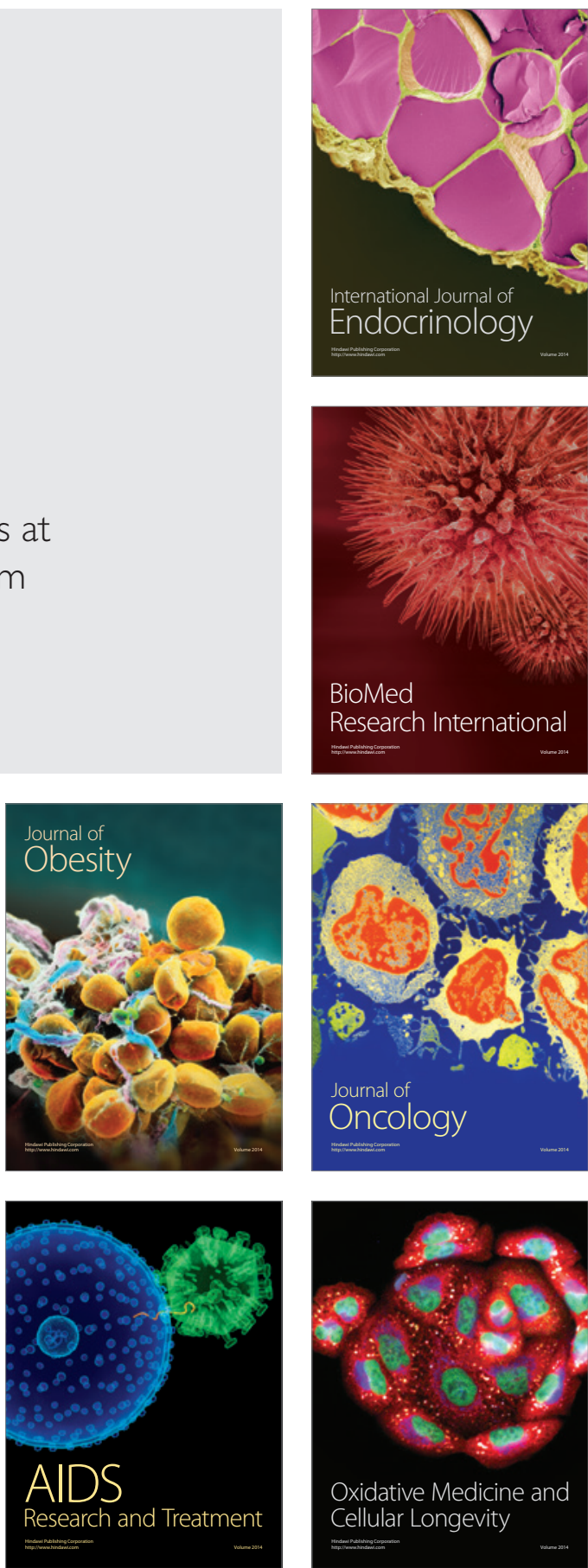